\title{
Effective lagrangians for QCD at high density
}

\author{
Roberto Casalbuoni \\ Dipartimento di Fisica dell' Universita' di Firenze and Sezione INFN, L.go E. Fermi 2, 50125 \\ Firenze, Italy.E-mail: casalbuoni@fi.infn.it
}

\begin{abstract}
We describe low energy physics in the CFL and LOFF phases by means of effective lagrangians. In the CFL case we present also how to derive expressions for the parameters appearing in the lagrangian via weak coupling calculations taking advantage of the dimensional reduction of fermion physics around the Fermi surface. The Goldstone boson of the LOFF phase turns out to be a phonon satisfying an anisotropic dispersion relation.
\end{abstract}

\section{INTRODUCTION}

Ideas about color superconductivity go back to almost 25 years ago [1], but only recently this phenomenon has received a lot of attention (for recent reviews see ref. [2]). The naive expectation is that at very high density, due to the asymptotic freedom, quarks would form a Fermi sphere of almost free fermions. However, Bardeen, Cooper and Schrieffer proved that the Fermi surface of free fermions is unstable in presence of an attractive, arbitrary small, interaction. Since in QCD the gluon exchange in the $\overline{3}$ channel is attractive one expects the formation of a coherent state of particle/hole pairs (Cooper pairs). For a careful description of the formation of the condensates and of the approximations involved in going from asymptotic densities to finite ones it is useful to see the contribution of K. Rajagopal at this meeting [3]. The phase structure of QCD at high density depends on the number of flavors and there are two very interesting cases, corresponding to two massless flavors $(2 \mathrm{SC})[1,4]$ and to three massless flavors (CFL) $[5,6]$ respectively. In this talk we will be mainly concerned with the latter case. The two cases correspond to very different patterns of symmetry breaking. If we denote leftand right-handed quark fields by $\psi_{i L(R)}^{\alpha}$ with $\alpha=1,2,3$, the $S U(3)_{c}$ color index, and $i=1, \cdots, N_{f}$ the flavor index ( $N_{f}$ is the number of massless flavors), in the 2SC phase we have the following structure for the condensate $\left(C=i \gamma^{2} \gamma^{0}\right.$ is the charge-conjugation matrix)

$$
\left\langle q_{i L(R)}^{\alpha} C q_{j L(R)}^{\beta}\right\rangle \propto \varepsilon_{i j} \varepsilon^{\alpha \beta 3} .
$$

The condensate breaks the color group $S U(3)_{c}$ down to the subgroup $S U(2)_{c}$ but it does not break any flavor symmetry. Although the baryon number, $B$, is broken, there is a combination of $B$ and of the broken color generator, $T_{8}$, which is unbroken in the $2 \mathrm{SC}$ phase. Therefore no massless Goldstone bosons are present in this phase. On the other hand, five gluon fields acquire mass whereas three are left massless. It is worth to notice that for the electric charge the situation is very similar to the one for the baryon number. Again a linear combination of the broken electric charge and of the broken generator 
$T_{8}$ is unbroken in the $2 \mathrm{SC}$ phase. The condensate (1) gives rise to a gap, $\Delta$, for quarks of color 1 and 2, whereas the two quarks of color 3 remain un-gapped (massless). The resulting effective low-energy theory has been described in [7]. In this contribution we will be mainly interested in the formulation of the effective theory for the three massless quarks case. At high density it has been shown that the following condensate is formed $[5,6]$

$$
\left\langle q_{i L(R)}^{\alpha} C q_{j L(R)}^{\beta}\right\rangle \propto \varepsilon^{i j X} \varepsilon_{\alpha \beta X}+\kappa\left(\delta_{\alpha}^{i} \delta_{\beta}^{j}+\delta_{\beta}^{i} \delta_{\alpha}^{j}\right)
$$

Due to the Fermi statistics, the condensate must be symmetric in color and flavor. As a consequence the two terms appearing in eq. (2) correspond to the $(\overline{\mathbf{3}}, \overline{\mathbf{3}})$ and $(\mathbf{6}, \mathbf{6})$ channels of $S U(3)_{c} \otimes S U(3)_{L(R)}$. It turns out that $\kappa$ is small $[5,8,9]$ and therefore the condensation occurs mainly in the $(\overline{\mathbf{3}}, \overline{\mathbf{3}})$ channel. The expression (2) shows that the ground state is left invariant by a simultaneous transformation of $S U(3)_{c}$ and $S U(3)_{L(R)}$. This is called Color Flavor Locking (CFL). The symmetry breaking pattern is

$$
\begin{gathered}
S U(3)_{c} \otimes S U(3)_{L} \otimes S U(3)_{R} \otimes U(1)_{B} \otimes U(1)_{A} \\
\downarrow \\
S U(3)_{c+L+R} \otimes Z_{2} \otimes Z_{2}
\end{gathered}
$$

The $U(1)_{A}$ symmetry is broken at the quantum level by the anomaly, but it gets restored at very high density since the instanton contribution is suppressed $[10,8,11]$. The $Z_{2}$ symmetries arise since the condensate is left invariant by a change of sign of the left- and/or right-handed fields. As for the 2SC case the electric charge is broken but a linear combination with the broken color generator $T_{8}$ annihilates the ground state. On the contrary the baryon number is broken. Therefore there are $8+2$ broken global symmetries giving rise to 10 Goldstone bosons. The one associated to $U(1)_{A}$ gets massless only at very high density. The color group is completely broken and all the gauge particles acquire mass. Also all the fermions are gapped. We will show in the following how to construct an effective lagrangian describing the Goldstone bosons, and how to compute their couplings in the high density limit where the QCD coupling gets weaker. A final problem we will discuss has to do with the fact that when quarks (in particular the strange quark) are massive, their chemical potentials cannot be all equal. This situation has been modeled out in [12]. If the Fermi surfaces of different flavors are too far apart, BCS pairing does not occur. However it might be favorable for different quarks to pair each of one lying at its own Fermi surface and originating a pair of nonzero total momentum. This is the LOFF state first studied by the authors of ref. [13] in the context of electron superconductivity in the presence of magnetic impurities. Since the Cooper pair has non-zero momentum the condensate breaks space symmetries and we will show that in the low-energy spectrum a massless particle, a phonon, the Goldstone boson of the broken translational symmetry, is present. We will construct the effective lagrangian also for this case. 


\section{EFFECTIVE THEORY FOR THE CFL PHASE}

We start introducing the Goldstone fields as the phases of the condensates in the $(\overline{\mathbf{3}}, \overline{\mathbf{3}})$ channel $[14,15]$

$$
X_{\alpha}^{i} \approx \varepsilon^{i j k} \varepsilon_{\alpha \beta \gamma}\left\langle q_{\beta L}^{j} q_{\gamma L}^{k}\right\rangle^{*}, \quad Y_{\alpha}^{i} \approx \varepsilon^{i j k} \varepsilon_{\alpha \beta \gamma}\left\langle q_{\beta R}^{j} q_{\gamma R}^{k}\right\rangle^{*} .
$$

Since quarks belong to the representation $(\mathbf{3}, \mathbf{3})$ of $S U(3)_{c} \otimes S U(3)_{L(R)}$ and transform under $U(1)_{B} \otimes U(1)_{A}$ according to

$$
q_{L} \rightarrow e^{i(\alpha+\beta)} q_{L}, \quad q_{R} \rightarrow e^{i(\alpha-\beta)} q_{R}, \quad e^{i \alpha} \in U(1)_{B}, \quad e^{i \beta} \in U(1)_{A},
$$

the transformation properties of the fields $X$ and $Y$ under the total symmetry group $G=S U(3)_{c} \otimes S U(3)_{L} \otimes S U(3)_{R} \otimes U(1)_{B} \otimes U(1)_{A}$ are $\left(g_{c} \in S U(3)_{c}, g_{L(R)} \in S U(3)_{L(R)}\right)$

$$
X \rightarrow g_{c} X g_{L}^{T} e^{-2 i(\alpha+\beta)}, \quad Y \rightarrow g_{c} Y g_{R}^{T} e^{-2 i(\alpha-\beta)} .
$$

The fields $X$ and $Y$ are $U(3)$ matrices and as such they describe $9+9=18$ fields. Eight of these fields are eaten up by the gauge bosons, producing eight massive gauge particles. Therefore we get the right number of Goldstone bosons, $10=18-10$. These fields correspond to the breaking of the global symmetries in $G$ (18 generators) to the symmetry group of the ground state $H=S U(3)_{c+L+R} \otimes Z_{2} \otimes Z_{2}$ (8 generators). For the following it is convenient to separate the $U(1)$ factors in $X$ and $Y$ defining fields belonging to $S U(3)$

$$
X=\hat{X} e^{2 i(\phi+\theta)}, \quad Y=\hat{Y} e^{2 i(\phi-\theta)}, \quad \hat{X}, \hat{Y} \in S U(3) .
$$

The fields $\phi$ and $\theta$ can also be described through the determinants of $X$ and $Y$

$$
d_{X}=\operatorname{det}(X)=e^{6 i(\phi+\theta)}, \quad d_{Y}=\operatorname{det}(Y)=e^{6 i(\phi-\theta)},
$$

The transformation properties under $G$ are

$$
\hat{X} \rightarrow g_{c} \hat{X} g_{L}^{T}, \quad \hat{Y} \rightarrow g_{c} \hat{Y} g_{R}^{T}, \quad \phi \rightarrow \phi-\alpha, \quad \theta \rightarrow \theta-\beta .
$$

The breaking of the global symmetry can be discussed in terms of gauge invariant fields given by $d_{X}, d_{Y}$ and

$$
\Sigma_{j}^{i}=\sum_{\alpha}\left(\hat{Y}_{\alpha}^{j}\right)^{*} \hat{X}_{\alpha}^{i} \rightarrow \Sigma=\hat{Y}^{\dagger} \hat{X}
$$

The $\Sigma$ field describes the 8 Goldstone bosons corresponding to the breaking of the chiral symmetry $S U(3)_{L} \otimes S U(3)_{R}$, as it is made clear by the transformation properties of $\Sigma^{T}$, $\Sigma^{T} \rightarrow g_{L} \Sigma^{T} g_{R}^{\dagger}$. That is $\Sigma^{T}$ transforms exactly as the usual chiral field. The other two fields $d_{X}$ and $d_{Y}$ provide the remaining two Goldstone bosons related to the breaking of the $U(1)$ factors.

In order to build up an invariant lagrangian, it is convenient to define the following currents

$$
J_{X}^{\mu}=\hat{X} D^{\mu} \hat{X}^{\dagger}=\hat{X}\left(\partial^{\mu} \hat{X}^{\dagger}+\hat{X}^{\dagger} g^{\mu}\right), \quad J_{Y}^{\mu}=\hat{Y} D^{\mu} \hat{Y}^{\dagger}=\hat{Y}\left(\partial^{\mu} \hat{Y}^{\dagger}+\hat{Y}^{\dagger} g^{\mu}\right),
$$


with $g_{\mu}=i g_{s} g_{\mu}^{a} T^{a} / 2$ the gluon field and $T^{a}=\lambda_{a} / 2$ the $S U(3)_{c}$ generators. These currents have simple transformation properties under the full symmetry group $G, J_{X, Y}^{\mu} \rightarrow$ $g_{c} J_{X, Y}^{\mu} g_{c}^{\dagger}$. The most general lagrangian, up to two derivative terms, invariant under $G$, the rotation group $O(3)$ (Lorentz invariance is broken by the chemical potential term) and the parity transformation defined as $\hat{X} \leftrightarrow \hat{Y}, \phi \rightarrow \phi, \theta \rightarrow-\theta$, is [14]

$$
\begin{aligned}
\mathcal{L}= & \left.\left.-\frac{F_{T}^{2}}{4} \operatorname{Tr}\left[\left(J_{X}^{0}-J_{Y}^{0}\right)^{2}\right)\right]-\alpha_{T} \frac{F_{T}^{2}}{4} \operatorname{Tr}\left[\left(J_{X}^{0}+J_{Y}^{0}\right)^{2}\right)\right]+\frac{1}{2}\left(\partial_{0} \phi\right)^{2}+\frac{1}{2}\left(\partial_{0} \theta\right)^{2} \\
& \left.\left.+\frac{F_{S}^{2}}{4} \operatorname{Tr}\left[\left(\vec{J}_{X}-\vec{J}_{Y}\right)^{2}\right)\right]+\alpha_{S} \frac{F_{S}^{2}}{4} \operatorname{Tr}\left[\left(\vec{J}_{X}+\vec{J}_{Y}\right)^{2}\right)\right]-\frac{v_{\phi}^{2}}{2}|\vec{\nabla} \phi|^{2}-\frac{v_{\theta}^{2}}{2}|\vec{\nabla} \theta|^{2} .
\end{aligned}
$$

Using $S U(3)_{c}$ color gauge invariance we can choose $\hat{X}=\hat{Y}^{\dagger}$, making 8 of the Goldstone bosons disappear and giving mass to the gluons. The properly normalized Goldstone bosons, $\Pi^{a}$, are given in this gauge by

$$
\hat{X}=\hat{Y}^{\dagger}=e^{i \Pi^{a} T^{a} / F_{T}},
$$

and expanding eq. (12) at the lowest order in the fields we get

$$
\mathcal{L} \approx \frac{1}{2}\left(\partial_{0} \Pi^{a}\right)^{2}+\frac{1}{2}\left(\partial_{0} \phi\right)^{2}+\frac{1}{2}\left(\partial_{0} \theta\right)^{2}-\frac{v^{2}}{2}\left|\vec{\nabla} \Pi^{a}\right|^{2}-\frac{v_{\phi}^{2}}{2}|\vec{\nabla} \phi|^{2}-\frac{v_{\theta}^{2}}{2}|\vec{\nabla} \theta|^{2}
$$

with $v=F_{S} / F_{T}$. The gluons $g_{0}^{a}$ and $g_{i}^{a}$ acquire Debye and Meissner masses given by

$$
m_{D}^{2}=\alpha_{T} g_{s}^{2} F_{T}^{2}, \quad m_{M}^{2}=\alpha_{S} v^{2} g_{s}^{2} F_{T}^{2} .
$$

It should be stressed that these are not the true rest masses of the gluons, since there is a large wave function renormalization effect making the gluon masses of order of the gap $\Delta$, rather than $\mu$ (see later) [16]. Since this description is supposed to be valid at low energies (we expect much below the gap $\Delta$ ), we could also decouple the gluons solving their classical equations of motion neglecting the kinetic term. The result from eq. (12) is

$$
g_{\mu}=-\frac{1}{2}\left(\hat{X} \partial_{\mu} \hat{X}^{\dagger}+\hat{Y} \partial_{\mu} \hat{Y}^{\dagger}\right)
$$

It is easy to show that substituting this expression in eq. (12) one gets [16]

$$
\mathcal{L}=\frac{F_{T}^{2}}{4}\left(\operatorname{Tr}\left[\dot{\Sigma} \dot{\Sigma}^{\dagger}\right]-v^{2} \operatorname{Tr}\left[\vec{\nabla} \Sigma \cdot \vec{\nabla} \Sigma^{\dagger}\right]\right)+\frac{1}{2}\left(\dot{\phi}^{2}-v_{\phi}^{2}|\vec{\nabla} \phi|^{2}\right)+\frac{1}{2}\left(\dot{\theta}^{2}-v_{\phi}^{2}|\vec{\nabla} \theta|^{2}\right) .
$$

Notice that the first term is nothing but the chiral lagrangian except for the breaking of the Lorentz invariance. This is a way of seeing the quark-hadron continuity, that is the continuity between the CFL and the nuclear matter phases in three flavor QCD. The identification is perfect if one realizes that in nuclear matter the pairing may occur in such a way to give rise to a superfluid due to the breaking of the baryon number as it happens in the CFL phase [17]. 


\section{FERMIONS NEAR THE FERMI SURFACE}

We will introduce now the formalism described in ref. [18] in order to evaluate the parameters appearing in the effective lagrangian. This formulation is based on the observation that, at very high-density, the energy spectrum of a massless fermion is described by states $| \pm\rangle$ with energies $E_{ \pm}=-\mu \pm \mid \vec{p}$ where $\mu$ is the quark number chemical potential. For energies much lower than the Fermi energy $\mu$, only the states $|+\rangle$ close to the Fermi surface. i.e. with $|\vec{p}| \approx \mu$, can be excited. On the contrary, the states $|-\rangle$ have $E_{-} \approx-2 \mu$ and therefore decouple. This can be seen more formally by writing the four-momentum of the fermion as

$$
p^{\mu}=\mu v^{\mu}+\ell^{\mu}
$$

where $v^{\mu}=\left(0, \vec{v}_{F}\right)$, and $\vec{v}_{F}$ is the Fermi velocity defined as $\vec{v}_{F}=\partial E /\left.\partial \vec{p}\right|_{\vec{p}=\vec{p}_{F}}$. For massless fermions $\left|\vec{v}_{F}\right|=1$. Since the hamiltonian for a massless Dirac fermion in a chemical potential $\mu$ is

$$
H=-\mu+\vec{\alpha} \cdot \vec{p}, \quad \vec{\alpha}=\gamma_{0} \vec{\gamma}
$$

one has

$$
H=-\mu\left(1-\vec{\alpha} \cdot \vec{v}_{F}\right)+\vec{\alpha} \cdot \vec{\ell} .
$$

Then, it is convenient to introduce the projection operators

$$
P_{ \pm}=\frac{1 \pm \vec{\alpha} \cdot \vec{v}_{F}}{2}
$$

such that

$$
H|+\rangle=\vec{\alpha} \cdot \vec{\ell}|+\rangle, \quad H|-\rangle=(-2 \mu+\vec{\alpha} \cdot \vec{\ell})|-\rangle .
$$

We can define fields corresponding to the states $| \pm\rangle$ through the decomposition

$$
\psi(x)=\sum_{\vec{v}_{F}} e^{-i \mu \nu \cdot x}\left[\psi_{+}(x)+\psi_{-}(x)\right],
$$

where an average over the Fermi velocity $\vec{v}_{F}$ is performed. The velocity-dependent fields $\psi_{ \pm}(x)$ are given by $\left(v^{\mu}=\left(0, \vec{v}_{F}\right)\right)$

$$
\psi_{ \pm}(x)=e^{i \mu \nu \cdot x}\left(\frac{1 \pm \vec{\alpha} \cdot \vec{v}_{F}}{2}\right) \psi(x)=\int_{|\ell|<\mu} \frac{d^{4} \ell}{(2 \pi)^{4}} e^{-i \ell \cdot x} \psi_{ \pm}(\ell) .
$$

Since we are interested at physics near the Fermi surface we integrate out all the modes with $|\ell|>\mu$. Substituting inside the Dirac part of the QCD lagrangian density one obtains $\left(V^{\mu}=\left(1, \vec{v}_{f}\right), \tilde{V}^{\mu}=\left(1,-\vec{v}_{F}\right)\right)$

$$
\mathcal{L}=\sum_{\vec{v}_{F}}\left[\psi_{+}^{\dagger} i V \cdot D \psi_{+}+\psi_{-}^{\dagger}(2 \mu+i \tilde{V} \cdot D) \psi_{-}+\left(\bar{\psi}_{+} i D_{\perp} \psi_{-}+\text {h.c. }\right)\right]
$$

where $\not_{\perp}=D_{\mu} \gamma_{\perp}^{\mu}$ and

$$
\gamma_{\perp}^{\mu}=\frac{1}{2} \gamma_{\nu}\left(2 g^{\mu \nu}-V^{\mu} \tilde{V}^{\nu}-\tilde{V}^{\mu} V^{v}\right)
$$


We notice that the fields appearing in this expression are evaluated at the same Fermi velocity because off-diagonal terms are canceled by the rapid oscillations of the exponential factor in the $\mu \rightarrow \infty$ limit. This behavior can be referred to as the Fermi velocity super-selection rule.

At the leading order in $1 / \mu$ one has

$$
i V \cdot D \psi_{+}=0, \quad \psi_{-}=-\frac{i}{2 \mu} \gamma_{0} \emptyset_{\perp} \psi_{+},
$$

showing the decoupling of $\psi_{-}$for $\mu \rightarrow \infty$. The equation for $\psi_{+}$shows also that only the energy and the momentum parallel to the Fermi velocity are relevant variables in the problem. We have an effective two-dimensional theory.

At the next to leading order the effective action for the field $\psi_{+}$is

$$
\mathcal{L}=\sum_{\vec{v}_{F}}\left[\psi_{+}^{\dagger} i V \cdot D \psi_{+}-\frac{1}{2 \mu} \psi_{+}^{\dagger}\left(D_{\perp}\right)^{2} \psi_{+}\right] .
$$

The previous remarks apply to any theory describing massless fermions at high density. The next step will be to couple this theory in a $S U(3)_{L} \otimes S U(3)_{R} \otimes S U(3)_{c}$ invariant way to Nambu-Goldstone bosons (NGB) describing the appropriate breaking for the CFL phase (we will not discuss here the determination of the parameters relevant for the $U(1)_{A, B}$ fields, see [11]). Using a gradient expansion we get an explicit expression for the decay coupling constant of the Nambu-Goldstone boson as well for their velocity.

The invariant coupling between fermions and Goldstone fields reproducing the symmetry breaking pattern of eq. (2) is proportional to

$$
\gamma_{1} \operatorname{Tr}\left[\psi_{L}^{T} \hat{X}^{\dagger}\right] C \operatorname{Tr}\left[\psi_{L} \hat{X}^{\dagger}\right]+\gamma_{2} \operatorname{Tr}\left[\psi_{L}^{T} C \hat{X}^{\dagger} \psi_{L} \hat{X}^{\dagger}\right]+\text { h.c. }
$$

with an analogous expression for the right-handed fields. Here the spinors are meant to be Dirac spinors. The trace is operating over the group indices of the spinors and of the Goldstone fields. Since the vacuum expectation value of the Goldstone fields is $\langle\hat{X}\rangle=\langle\hat{Y}\rangle=1$, we see that this coupling induces the right breaking of the symmetry. In the following we will consider only the case $\gamma_{2}=-\gamma_{1} \propto \Delta / 2$, where $\Delta$ is the gap parameter.

Since the transformation properties under the symmetry group of the fields at fixed Fermi velocity do not differ from those of the quark fields, for both left-handed and right-handed fields we get the effective lagrangian density

$$
\begin{aligned}
\mathcal{L} & =\sum_{\vec{v}_{F}} \frac{1}{2}\left[\sum_{A=1}^{9}\left(\psi_{+}^{A \dagger} i V \cdot D \psi_{+}^{A}+\psi_{-}^{A \dagger} i \tilde{V} \cdot D \psi_{-}^{A}-\Delta_{A}\left(\psi_{-}^{A} C \psi_{+}^{A}+\text { h.c. }\right)\right)\right. \\
& \left.-\Delta \sum_{I=1,3}\left(\operatorname{Tr}\left[\left(\psi_{-} X_{1}^{\dagger}\right)^{T} C \varepsilon_{I}\left(\psi_{+} X_{1}^{\dagger}\right) \varepsilon_{I}\right]+\text { h.c. }\right)\right],
\end{aligned}
$$

where we have introduced the fields $\psi_{ \pm}^{A}$ :

$$
\psi_{ \pm}=\frac{1}{\sqrt{2}} \sum_{A=1}^{9} \lambda_{A} \psi_{ \pm}^{A} .
$$


Here $\lambda_{a}(a=1, \ldots, 8)$ are the Gell-Mann matrices normalized as follows: $\operatorname{Tr}\left(\lambda_{a} \lambda_{b}\right)=$ $2 \delta_{a b}$ and $\lambda_{9}=\sqrt{2 / 3} 1$. Furthermore $\Delta_{1}=\cdots=\Delta_{8}=\Delta, \Delta_{9}=-2 \Delta$, and $X_{1}=\hat{X}-1$. Notice that the NGB fields couple to fermionic fields with opposite Fermi velocities. In this expression, as in the following ones, the field $\psi_{-}$is defined as $\psi_{+}$with $\vec{v}_{F} \rightarrow-\vec{v}_{F}$, and therefore it is not the same as the one defined in (24).

The formalism becomes more compact by introducing the Nambu-Gorkov fields

$$
\chi=\left(\begin{array}{c}
\psi_{+} \\
C \psi_{-}^{*}
\end{array}\right)
$$

It is important to realize that the fields $\chi$ and $\chi^{\dagger}$ are not independent variables. In fact, since we integrate over all the Fermi surface, the fields $\psi_{-}^{*}$ and $\psi_{+}$, appearing in $\chi$, appear also in $\chi^{\dagger}$ with $\vec{v}_{F} \rightarrow-\vec{v}_{F}$. In order to avoid this problem we can integrate over half of the Fermi surface, or, taking into account the invariance under $\vec{v}_{F} \rightarrow-\vec{v}_{F}$, we can simply integrate over all the sphere with a weight $1 / 8 \pi$ instead of $1 / 4 \pi$. Then the first three terms in the lagrangian density (30) become

$$
\mathcal{L}_{0}=\int \frac{d \vec{v}_{F}}{8 \pi} \frac{1}{2} \sum_{A=1}^{9} \chi^{A \dagger}\left[\begin{array}{cc}
i V \cdot D & \Delta^{A} \\
\Delta^{A} & i \tilde{V} \cdot D^{*}
\end{array}\right] \chi^{A},
$$

so that, in momentum space the free fermion propagator is

$$
S_{A B}(p)=\frac{2 \delta_{A B}}{V \cdot p \tilde{V} \cdot p-\Delta_{A}^{2}}\left[\begin{array}{cc}
\tilde{V} \cdot p & -\Delta_{A} \\
-\Delta_{A} & V \cdot p
\end{array}\right] .
$$

We are now in position to evaluate the self-energy of the Goldstone bosons through their coupling to the fermions at the Fermi surface. There are two one-loop contributions [16], one from the coupling $\Pi \chi \chi$ and a tadpole from the coupling $\Pi \Pi \chi \chi$ (see eq. (30). The tadpole diagram contributes only to the mass term and it is essential to cancel the external momentum independent term arising from the other diagram. Therefore, as expected, the mass of the NGB's is zero. The contribution at the second order in the momentum expansion is given by

$$
i \frac{21-8 \ln 2}{72 \pi^{2} F_{T}^{2}} \int \frac{d \vec{v}_{F}}{4 \pi} \sum_{a=1}^{8} \Pi^{a} V \cdot p \tilde{V} \cdot p \Pi^{a} .
$$

Integrating over the velocities and going back to the coordinate space we get

$$
\mathcal{L}_{\mathrm{eff}}^{\mathrm{kin}}=\frac{21-8 \ln 2}{72 \pi^{2} F_{T}^{2}} \sum_{a=1}^{8}\left(\dot{\Pi}^{a} \dot{\Pi}^{a}-\frac{1}{3}\left|\vec{\nabla} \Pi_{a}\right|^{2}\right) .
$$

We can now determine the decay coupling constant $F_{T}$ through the requirement of getting the canonical normalization for the kinetic term; this implies

$$
F_{T}^{2}=\frac{\mu^{2}(21-8 \ln 2)}{36 \pi^{2}}
$$


a result obtained by many authors using different methods (for a complete list of the relevant papers see the first reference of [2]). We see also that $v^{2}=1 / 3$. The other constants appearing in our effective lagrangian can be obtained via a direct calculation of $m_{D}$ and $m_{M}$ [16]. This is done evaluating the one-loop contribution to the gluon self-energy. Also in this case there are two contributions, one coming from the gauge coupling to the fermions, whereas the other arises from the next-to leading (in $\mu$ ) seagull contribution to the fermion effective lagrangian in eq. (28). The results we find are $[16,11]$

$$
m_{D}^{2}=g_{s}^{2} F_{T}^{2}, \quad m_{M}^{2}=\frac{1}{3} m_{D}^{2}
$$

Comparison with equation (15) shows that

$$
\alpha_{S}=\alpha_{T}=1
$$

Performing a gradient expansion of the gluon self-energy one finds that there is a wave function renormalization of order $g_{s} \mu / \Delta \gg 1$. Extrapolating this result, the physical masses of the gluons turn out to be of the order of the gap energy $(\approx 1.70 \Delta)$ [16]. The origin of the pion velocity $1 / \sqrt{3}$ is a direct consequence of the integration over the Fermi velocity. Therefore it is completely general and applies to all the NGB's in the theory, including the ones associated to the breaking of $U(1)_{V}$ and $U(1)_{A}\left(v_{\phi}^{2}=v_{\theta}^{2}=1 / 3\right)$; needless to say that higher order terms in the expansion $1 / \mu$ could change this result.

The breaking of the Lorentz invariance exhibited by the pion velocity different from one, can be seen also in the matrix element $\left\langle 0\left|J_{\mu}^{a}\right| \Pi^{b}\right\rangle$. Its evaluation gives [16]

$$
\left\langle 0\left|J_{\mu}^{a}\right| \Pi^{b}\right\rangle=i F_{T} \delta_{a b} \tilde{p}_{\mu}, \quad \tilde{p}^{\mu}=\left(p^{0}, \vec{p} / 3\right) .
$$

The current is conserved, as a consequence of the dispersion relation satisfied by NGB's.

\section{THE LOFF PHASE}

We shall now consider massless quarks of three colors and two different flavors. At finite densities we introduce two chemical potentials, $\mu_{1}$ and $\mu_{2}$, for the two species in order to mimic the different mass case. We write

$$
\mu_{1}-\mu_{2}=\delta \mu \ll \mu=\frac{\mu_{1}+\mu_{2}}{2} .
$$

The BCS condensation takes place also for $\delta \mu \neq 0$ provided $\delta \mu \ll \Delta$. On the other hand, for $\delta \mu \approx \Delta$, the picture changes significantly. The analysis in [12] shows that there exist two values of $\delta \mu, \delta \mu_{1}$ and $\delta \mu_{2}$, such that, for $\delta \mu \in\left(\delta \mu_{1}, \delta \mu_{2}\right)$ the high density quarkgluon matter is in a phase characterized by the breaking of translational and rotational invariance, due to the presence of a scalar and a vector condensate. This phenomenon is called crystalline color superconductivity of QCD and the relative phase is named LOFF phase. The authors of ref. [12] find $\delta \mu_{1}=0.71 \Delta$ and $\delta \mu_{2}=0.744 \Delta$ for $\mu=0.4 \mathrm{GeV}$ and $\Delta=40 \mathrm{MeV}$ for a point-like four-fermi coupling. More recently it has been found that in the one-gluon exchange approximation the window opens up considerably [19]. 
The condensation in the LOFF phase gives rise to two breaking terms in the fermion lagrangian characterized by two gap parameters $\Delta^{(s)}$ and $\Delta^{(v)}$

$$
-\frac{1}{2} e^{2 i \vec{q} \cdot \vec{x}} \sum_{\vec{v}_{F}} e^{i \delta \mu \vec{v}_{F} \cdot \vec{x}}\left[\Delta^{(s)} \varepsilon_{i j}+\vec{v} \cdot \vec{n} \Delta^{(v)} \sigma_{i j}^{1}\right] \varepsilon^{\alpha \beta 3} \psi_{+\vec{v} ; i \alpha} C \psi_{-\vec{v} ; j \beta}-(L \rightarrow R),
$$

with $\vec{n}=\vec{q} /|\vec{q}|$. The condensates break the space symmetry group. However the discussion of the number of NGB's in the case of space symmetries is a subtle one due to the particular group structure. In fact rotations and translations cannot be considered transformations breaking the symmetries of the theory in an independent way. This is because a translation plus a rotation is physically equivalent to a translation. Let us discuss the consequences of this situation more closely.

We first consider spatial rotations. We define a vector field $\vec{R}(x)$ such that $|\vec{R}|^{2}=1$ and $\langle\vec{R}\rangle_{0}=\vec{n}$ [20]. The rotational symmetry is restored by substituting $\vec{v} \cdot \vec{n} \rightarrow \vec{v} \cdot \vec{R}$ in the term proportional to $\Delta^{(v)}$. Let us now consider the exponential factor $\exp (2 i \vec{q} \cdot \vec{x})$ in (42), which breaks both rotational and translational invariance. By introducing a field $\Phi(x)$ behaving as a scalar under the space group [20], we restore translational and rotational invariance via the substitution $2 \vec{q} \cdot \vec{x} \rightarrow \Phi(x)$. We assume $\langle\Phi(x)\rangle_{0}=2 \vec{q} \cdot \vec{x}$. We then introduce a field $\phi(x)$ through

$$
\Phi(x)=2 \vec{q} \cdot \vec{x}+\phi(x), \quad\langle\phi(x)\rangle_{0}=0
$$

and convenient transformation properties such to compensate the variation of the term $\vec{q} \cdot \vec{x}$ under the space group [20]. The field $\phi$ acts as the phonon (Nambu-Goldstone boson) field associated to the breaking of the space symmetry. We can now construct the field $\vec{R}$ in terms of $\Phi(x)$ as $\vec{R}=\vec{\nabla} \Phi /|\vec{\nabla} \Phi|$. This expression satisfies the required properties for $\vec{R}(x)$.

Through a bosonization procedure similar to the one employed in the previous Section, one can derive an effective lagrangian for the NGB field. The effective lagrangian must contain only derivative terms. Polynomial terms are indeed forbidden by translation invariance, since $\phi$ is not a scalar field under space transformations. In order to write the kinetic terms is better to use the field $\Phi$ which behaves as a scalar under both rotations and translation. However since the expectation value of the gradient of $\Phi$ is given by $\langle\vec{\nabla} \Phi\rangle_{0}=2 \vec{q} \approx \Delta$, we cannot limit the expansion in the spatial derivatives of $\Phi$ to any finite order. A real spatial derivative expansion can be made only for the phonon field $\phi$. With this in mind the most general invariant lagrangian will contain a tower of space-derivative terms [20]:

$$
\mathcal{L}\left(\phi, \partial_{\mu} \phi\right)=\frac{f^{2}}{2}\left[\dot{\Phi}^{2}-\sum_{n=1}^{\infty} c_{n}\left(|\vec{\nabla} \Phi|^{2}\right)^{n}\right]
$$

Here $\Phi$ must be thought as a function of the phonon field $\phi$. Using

$$
|\vec{\nabla} \Phi(x)|^{2}=4 q^{2}+\frac{4 q}{f} \vec{n} \cdot \vec{\nabla} \phi(x)+\frac{1}{f^{2}}|\vec{\nabla} \phi(x)|^{2},
$$


at the lowest order in the derivatives of the phonon field $\phi$ we get (neglecting a constant term):

$$
\mathcal{L}\left(\phi, \partial_{\mu} \phi\right)=\frac{1}{2}\left[\dot{\phi}^{2}-v_{\|}^{2}\left|\vec{\nabla}_{\|} \phi\right|^{2}-v^{2}\left(4 q f \vec{\nabla}_{\|} \phi+|\vec{\nabla} \phi|^{2}\right)\right],
$$

where $\vec{\nabla}_{\|} \phi=\vec{n} \cdot \vec{\nabla} \phi$ and $v_{\|}^{2}, v^{2}$ are constants. Notice that the linear term gives rise to a surface contribution. The lack of rotational invariance in (46) follows from the gradient expansion due to the non-linear transformations undergone by the field $\phi(x)$. This happens also in the analogous expansion for the chiral field. Therefore the physical consequence of the extraction of the expectation value of $\Phi$ is an anisotropy in the dispersion relation for the phonon field $\phi(x)$.

\section{ACKNOWLEDGMENTS}

I would like to thank R. Gatto, M. Mannarelli and G. Nardulli for their precious collaboration to the papers originating this contribution.

\section{REFERENCES}

1. B. Barrois, Nuclear Physics B129, 390 (1977); S. Frautschi, Proceedings of workshop on hadronic matter at extreme density, Erice 1978; D. Bailin and A. Love, Physics Report 107, 325 (1984).

2. K. Rajagopal and F. Wilczek, hep-ph/0011333; S.D.H. Hsu, hep-ph/0003140; D.K. Hong, hepph/0101025; M. Alford, hep-ph/0102047.

3. K. Rajagopal, contributed paper to this meeting.

4. M. Alford, K. Rajagopal and F. Wilczek, Physics Letters B422, 247 (1998), hep-ph/9711395; R. Rapp, T. Schäfer, E.V. Shuryak and M. Velkovsky, Physical Review Letters 81, 53 (1998), hep$\mathrm{ph} / 9711396$.

5. M. Alford, K. Rajagopal and F. Wilczek, Nuclear Physics B537, 443 (1999), hep-ph/9804403.

6. T. Schäfer and F. Wilczek, Physical Review Letters 82, 3956 (1999), hep-ph/9811473.

7. R. Casalbuoni, Z. Duan and F. Sannino, Physical Review D62, 094004 (2000), hep-ph/0004207; ibidem D63, 114026 (2001), hep-ph/0011394.

8. T. Schäfer, Nuclear Physics B575, 269 (2000), hep-ph/9909574.

9. I.A. Shovkovy and L.C. Wijewardhana, Physics Letters B470, 189 (1999), hep-ph/9910225.

10. R. Rapp, T. Schäfer, E.V. Shuryak and M. Velkovsky, Annals of Physics 280, 35 (2000), hep$\mathrm{ph} / 9904353$.

11. D.T. Son and M.A. Stephanov, Physical Review D61, 074012 (2000), hep-ph/9910491; ibidem Erratum D62, 059902 (2000), hep-ph/0004095.

12. M. Alford, J.A. Bowers and K. Rajagopal, Physical Review D63, 074016 (2001), hep-ph/0008208.

13. A. Larkin and Y.N. Ovchinnikov, Soviet Physics JETP 20, 762 (1965); P. Fulde and R.A. Ferrel, Physical Review 135A, 550 (1964).

14. R. Casalbuoni and R. Gatto, Physics Letters B464, 111 (1999), hep-ph/9908227.

15. D.K. Hong, M. Rho and I. Zahed, Physics Letters B468, 261 (1999), hep-ph/9906551.

16. R. Casalbuoni, R. Gatto and G. Nardulli, Physics Letters B498, 179 (2001), hep-ph/0010321.

17. T.Schäfer and F. Wilczek, Physical Review Letters 82, 3956 (1999), hep-ph/9811473.

18. D.K. Hong, Physics Letters B473, 118 (2000), hep-ph/9812510; D.K. Hong Nuclear Physics B582, 451 (2000), hep-ph/9905523; S.R. Beane, P.F. Bedaque and M.J. Savage, Physics Letters B483, 131 (2000), hep-ph/0002209.

19. A.K. Leibovich, K. Rajagopal and E. Shuster, hep-ph/0104073.

20. R. Casalbuoni, R. Gatto, M. Mannarelli and G. Nardulli, Physics Letters B511, 218 (2001), hep$\mathrm{ph} / 0101326$. 